\title{
HIGHLIGHTS
}

LUPUS NEPHRITIS

\section{Sustained proteinuria and dyslipidemia are risk factors for progressive CKD in patients with lupus nephritis}

Not all patients with systemic lupus erythematosus (SLE) are at increased risk of developing chronic kidney disease (CKD), new research finds. In this Canadian study, patients with SLE but without nephritis had similar rates of decline in renal function to non-SLE populations. In patients with lupus nephritis, however, the rate of CKD progression was increased and linked with clinical abnormalities. In their article, the researchers highlight sustained proteinuria and high cholesterol levels as important interrelated and modifiable risk factors for progressive loss of kidney function in this subgroup.

Heather Reich and co-workers analyzed an electronic database of clinical records for 504 patients with SLE (mean age 35 years, average follow-up 11 years), of whom 266 developed lupus nephritis. "We wanted to clarify what happens to kidney function in patients with SLE, determine how many of these patients develop CKD, and examine risk factors that may predict renal function decline," states Reich.

All patients who developed CKD during

follow-up had some pre-existing renal impairment, but only 28 (11.8\%) of 238 patients without nephritis developed CKD. These patients were older (mean age 54 years) and had slowly declining renal function. By contrast, 63 (24\%) of the 266 patients with nephritis developed CKD. These patients (mean age 39 years) showed an accelerated decline in renal function (estimated glomerular filtration rate decline $-4.35 \mathrm{ml} / \mathrm{min} / 1.73 \mathrm{~m}^{2}$ per year in patients with nephritis versus $-0.66 \mathrm{ml} /$ $\mathrm{min} / 1.73 \mathrm{~m}^{2}$ per year in those without) and six patients developed end-stage renal disease needing renal replacement therapy.

This study dispels some of the myths about the risks for progressive CKD in SLE. "We were really surprised to find that SLE itself isn't a risk factor for progressive $\mathrm{CKD}$, especially when the prevailing hypothesis has always been that background systemic inflammation is a major risk factor," Reich explains. "Patients who develop progressive CKD have early and usually modifiable risk factors; the key now is to refine our ability to identify which patients are at risk."

\section{This study dispels some of} the myths about the risks for progressive CKD in SLE 77

Anticipated results from ongoing clinical trials, on prophylactic statins and other interventions in SLE, may prove to be useful for defining treatment paradigms that balance the risks of therapy with preventing progressive CKD.

\section{Steven E. Bradshaw}

Original article Reich, H. N. et al. Persistent proteinuria and dyslipidemia increase the risk of progressive chronic kidney disease in lupus erythematosus. Kidney Int. doi:10.1038/ ki.2010.525 